\title{
Comparable nonlinear contractions in ordered metric spaces
}

\author{
Aftab Alam*, Qamrul Haq Khan, Mohammad Imdad \\ Department of Mathematics, Aligarh Muslim University, Aligarh-202002, India. \\ Communicated by Z. Kadelburg
}

\begin{abstract}
In this article, we generalize some frequently used metrical notions such as: completeness, closedness, continuity, gcontinuity and compatibility to order-theoretic setting especially in ordered metric spaces and utilize these relatively weaker notions to prove some existence and uniqueness results on coincidence points for $\mathrm{g}$-comparable mappings satisfying Boyd-Wong type nonlinear contractivity conditions. We also furnish some illustrative examples to demonstrate our results. Finally, as an application of our certain newly proved results, we establish the existence and uniqueness of solution of an integral equation. (C)2017 All rights reserved
\end{abstract}

Keywords: Ordered metric space, TCC property, g-comparable mappings, g-admissible mappings, termwise monotone sequence.

2010 MSC: 47H10, 54H25.

\section{Introduction}

The classical Banach contraction principle and its applications are well-known. It has so many different generalizations with different approaches. One of the remarkable generalizations, known as $\varphi$ contraction, was given by Browder [10] in 1968, wherein author assumed $\varphi$ to be right continuous and increasing control function and utilized the same to generalize Banach contraction principle. Later, many authors generalized Browder's fixed point theorem by varying the properties of control function $\varphi$. In 1969, Boyd and Wong [9] observed that it is sufficient to assume merely the right-upper semicontinuity of $\varphi$ (without monotonicity requirement on $\varphi$ ) and extended Browder's fixed point theorem by introducing the following family of control functions:

$$
\Psi=\{\varphi:[0, \infty) \rightarrow[0, \infty): \varphi(t)<t \text { for each } t>0 \text { and } \varphi \text { is right }- \text { upper semicontinuous }\} .
$$

Inspired by Boyd and Wong [9], in 1977, Mukherjea [24] slightly modified Browder's fixed point theorem by introducing the following family of control functions:

$$
\Theta=\{\varphi:[0, \infty) \rightarrow[0, \infty): \varphi(t)<t \text { for each } t>0 \text { and } \varphi \text { is right continuous }\} .
$$

\footnotetext{
${ }^{*}$ Corresponding author

Email addresses: aafu. amu@gmail.com (Aftab Alam), qhkhan.ssitm@gmail.com (Qamrul Haq Khan), mhimdad@yahoo.co.in (Mohammad Imdad)
}

doi:10.22436/jnsa.010.04.29 
The following family of control functions available in the existing literature is more natural:

$$
\mathfrak{I}=\{\varphi:[0, \infty) \rightarrow[0, \infty): \varphi(t)<t \text { for each } \mathrm{t}>0 \text { and } \varphi \text { is continuous }\} .
$$

The following family of control functions is essentially due to Lakshmikantham and Ćirić [23]:

$$
\Phi=\left\{\varphi:[0, \infty) \rightarrow[0, \infty): \varphi(t)<\mathrm{t} \text { for each } \mathrm{t}>0 \text { and } \lim _{\mathrm{r} \rightarrow \mathrm{t}^{+}} \varphi(\mathrm{r})<\mathrm{t} \text { for each } \mathrm{t}>0\right\} .
$$

The following family of control functions is obtained in Boyd and Wong [9] which was later utilized in Jotic [19]:

$$
\Omega=\left\{\varphi:[0, \infty) \rightarrow[0, \infty): \varphi(t)<t \text { for each } t>0 \text { and } \limsup _{r \rightarrow t^{+}} \varphi(r)<t \text { for each } t>0\right\} .
$$

Recently, Alam et al. [4] studied the following relation among earlier described classes of control functions.

Proposition 1.1 ([4]). The class $\Omega$ enlarges the classes $\Psi, \Theta, \mathfrak{I}$ and $\Phi$ under the following inclusion relation:

$$
\mathfrak{I} \subset \Theta \subset \Psi \subset \Omega, \text { and } \mathfrak{I} \subset \Theta \subset \Phi \subset \Omega \text {. }
$$

Throughout the manuscript, $\mathbb{N}_{0}$ denotes the set of nonnegative integers (i.e., $\mathbb{N}_{0}:=\mathbb{N} \cup\{0\}$ ). In what follows, by the pair $(X, \preceq)$, we mean a nonempty set $X$ equipped with a partial order $\preceq$, often called an ordered set. We denote $\succeq$ by the dual order of $\preceq$ (i.e. $x \succeq y$ means $y \preceq x$ ). Two elements $x$ and $y$ in an ordered set $(X, \preceq)$ are said to be comparable if either $x \preceq y$ or $y \preceq x$ and we denote it as: $x \prec \succ y$. A subset $E$ of an ordered set is called totally ordered if $x \prec \succ y$ for all $x, y \in E$. For a pair of self-mappings $f$ and $g$ defined on an ordered set $(X, \preceq)$, we say that $f$ is $g$-increasing (resp. $g$-decreasing), if for any $x, y \in X g(x) \preceq g(y) \Rightarrow f(x) \preceq f(y)$ (resp. $f(x) \succeq f(y))$. Also, $f$ is called $g$-monotone if $f$ is either $g$ increasing or $g$-decreasing. Further, under the restriction $g=I$, the identity mapping on $X$, the notions of $g$-increasing, g-decreasing and $g$-monotone mappings respectively reduce to increasing, decreasing and monotone mappings. Following $\mathrm{O}^{\prime}$ Regan and Petruşel [28], the triplet $(X, d, \preceq)$ is called ordered metric space wherein a nonempty set $X$ is equipped with a metric $d$ and a partial order $\preceq$. If, in addition, $d$ is a complete metric on $X$, then we say that $(X, d, \preceq)$ is ordered complete metric space.

In the last decades, there has been a growing interest in studying the existence of fixed points for monotone contractive mappings in ordered metric spaces. This trend was initiated by Turinici [32, 33]. Later, Ran and Reurings [29] proved a slightly more natural version of the corresponding fixed point theorems of Turinici (cf. [32,33]) for continuous monotone mappings with some applications to matrix equations. In subsequent papers many authors extended and refined the fixed point theorems of Ran and Reurings [29] and proved various fixed point theorems in ordered metric spaces (e.g. [1, 3, 4, 6, 7, 11, 12, $15-18,25,26,28,37])$. All such results involve some variant of monotone mappings.

To avoid the necessity of monotonicity, several authors like as: Nieto and Rodríguez-López [27], Turinici [34, 35] and Dorić et al. [13] assumed the property that the underlying mapping maps comparable elements to comparable elements, which is relatively weaker than monotonicity requirement on the mapping and often easy to check. Recently, Alam and Imdad [2] termed such mapping as comparable mappings and also generalized this idea for a pair of mappings (namely: $f$ and $g$ ) by introducing the notion of $g$-comparable mappings. Utilizing the notion of $g$-comparability, Alam and Imdad [2] proved some coincidence theorems under linear contractions without using g-monotonicity in two different directions, namely either the underlying metric space $X$ or the range subspaces $(f(X)$ or $g(X))$ are complete.

The aim of this paper is to define $\prec \succ$-analogues of some metrical notions (such as: completeness, closedness, continuity, g-continuity and compatibility) and utilizing these notions to extend the coincidence theorem under nonlinear contractivity condition due to Boyd and Wong [9]. Particularly, we observe that in our results neither the whole space $X$ nor the range subspaces $(f(X)$ or $g(X))$ are required to be necessarily complete. 


\section{Preliminaries and auxiliary results}

In this section, we collect some basic notions and auxiliary results needed in our subsequent discussions.

Definition $2.1([5])$. Let $(X, d, \preceq)$ be an ordered metric space and $Y$ a nonempty subset of $X$. Then $d$ and $\preceq$ respectively induce a metric $d_{Y}$ and a partial order $\preceq_{Y}$ on $Y$ so that

$$
\mathrm{d}_{Y}(x, y)=d(x, y), \quad \forall x, y \in Y,
$$

and

$$
x \preceq Y y \Leftrightarrow x \preceq y, \quad \forall x, y \in Y .
$$

Thus $\left(Y, d_{Y}, \preceq_{Y}\right)$ is an ordered metric space, which is called a subspace of $(X, d, \preceq)$.

Conventionally, we opt to refer $Y$ as a subspace of $X$ rather than saying $\left(Y, d_{Y}, \preceq_{Y}\right)$ a subspace of $(X, d, \preceq)$ and continue to write $d$ and $\preceq$ instead of $d_{Y}$ and $\preceq_{Y}$ respectively.

Definition $2.2([20,22])$. Let $X$ be a nonempty set and $f$ and $g$ two self-mappings on $X$. Then,

(i) an element $x \in X$ is called a coincidence point of $f$ and $g$, if

$$
g(x)=f(x) ;
$$

(ii) if $x \in X$ is a coincidence point of $f$ and $g$ and $\bar{x} \in X$ such that $\bar{x}=g(x)=f(x)$, then $\bar{x}$ is called a point of coincidence of $f$ and $g$;

(iii) if $x \in X$ is a coincidence point of $f$ and g such that $x=g(x)=f(x)$, then $x$ is called a common fixed point of $f$ and $g$;

(iv) $f$ and $g$ are said to be commuting, if

$$
g(f x)=f(g x), \quad \forall x \in X ;
$$

(v) $f$ and $g$ are said to be weakly compatible (or partially commuting or coincidentally commuting), if $f$ and $g$ commute at their coincidence points, i.e., for any $x \in X$,

$$
g(x)=f(x) \Rightarrow g(f x)=f(g x) .
$$

Definition $2.3([21,31])$. Let $(X, d)$ be a metric space and $f$ and $g$ two self-mappings on $X$. Then

(i) $f$ and $g$ are said to be weakly commuting, if

$$
\mathrm{d}(\mathrm{gfx}, \mathrm{fg} x) \leqslant \mathrm{d}(\mathrm{g} x, \mathrm{fx}), \quad \forall x \in X ;
$$

(ii) $f$ and $g$ are said to be compatible if for any sequence $\left\{x_{n}\right\} \subset X$ and for any $z \in X$,

$$
\lim _{n \rightarrow \infty} g\left(x_{n}\right)=\lim _{n \rightarrow \infty} f\left(x_{n}\right)=z \Rightarrow \lim _{n \rightarrow \infty} d\left(g f x_{n}, f g x_{n}\right)=0 .
$$

Definition 2.4 ([30]). Let $(X, d)$ be a metric space, $f$ and $g$ two self-mappings on $X$ and $x \in X$. We say that $f$ is $g$-continuous at $x$, if for any sequence $\left\{x_{n}\right\} \subset X$,

$$
g\left(x_{n}\right) \stackrel{d}{\longrightarrow} g(x) \Rightarrow f\left(x_{n}\right) \stackrel{d}{\longrightarrow} f(x) .
$$

Moreover, $f$ is called $g$-continuous, if it is $g$-continuous at each point of $X$.

Notice that particularly, at $\mathrm{g}=\mathrm{I}$, the identity mapping on $\mathrm{X}$, Definition 2.4 reduces to the definition of continuity.

Now, we introduce the following notion. 
Definition 2.5. Let $f$ and $g$ be two self-mappings defined on a nonempty set $X$. We say that $f$ is $g$ admissible if for any $x, y \in X$,

$$
g(x)=g(y) \Rightarrow f(x)=f(y) .
$$

In the following line, we propose a characterization of $\mathrm{g}$-monotone mappings.

Proposition 2.6. Let $\mathrm{f}$ and $\mathrm{g}$ be two self-mappings defined on an ordered set $(\mathrm{X}, \preceq)$. If $\mathrm{f}$ is $\mathrm{g}$-monotone, then $\mathrm{f}$ is g-admissible.

Proof. Take $x, y \in X$ such that $g(x)=g(y)$. On using reflexivity of $\preceq$, we have

$$
g(x) \preceq g(y), \quad \text { and } \quad g(x) \succeq g(y) .
$$

Suppose that $f$ is $g$-increasing (resp. g-decreasing), we have

$$
f(x) \preceq f(y), \quad \text { and } \quad f(x) \succeq f(y) \quad(\text { resp. } f(x) \succeq f(y), \quad \text { and } \quad f(x) \preceq f(y)),
$$

which, in both the cases (owing to anti-symmetric property of $\preceq$ ) gives rise that

$$
f(x)=f(y) .
$$

Hence $f$ is $g$-admissible.

Finally we record the following known results needed in the proof of our main result.

Lemma 2.7 ([4]). Let $\varphi \in \Omega$. If $\left\{a_{n}\right\} \subset(0, \infty)$ is a sequence such that $a_{n+1} \leqslant \varphi\left(a_{n}\right)$, for all $n \in \mathbb{N}_{0}$, then $\lim _{n \rightarrow \infty} a_{n}=0$.

Lemma $2.8([8,18,36])$. Let $(X, d)$ be a metric space and $\left\{x_{n}\right\}$ a sequence in $X$. If $\left\{x_{n}\right\}$ is not a Cauchy sequence, then there exist $\epsilon>0$ and two subsequences $\left\{x_{n_{k}}\right\}$ and $\left\{x_{m_{k}}\right\}$ of $\left\{x_{n}\right\}$ such that

(i) $k \leqslant m_{k}<n_{k} \forall k \in \mathbb{N}$;

(ii) $\mathrm{d}\left(\mathrm{x}_{\mathrm{m}_{\mathrm{k}}}, \mathrm{x}_{\mathrm{n}_{\mathrm{k}}}\right)>\epsilon \forall \mathrm{k} \in \mathbb{N}$;

(iii) $\mathrm{d}\left(\mathrm{x}_{\mathrm{m}_{\mathrm{k}}}, \mathrm{x}_{\mathrm{n}_{\mathrm{k}-1}}\right) \leqslant \epsilon \forall \mathrm{k} \in \mathbb{N}$.

Moreover, suppose that $\lim _{n \rightarrow \infty} d\left(x_{n}, x_{n+1}\right)=0$, then

(iv) $\lim _{k \rightarrow \infty} d\left(x_{m_{k}}, x_{n_{k}}\right)=\epsilon$;

(v) $\lim _{k \rightarrow \infty} d\left(x_{m_{k}+1}, x_{n_{k}+1}\right)=\epsilon$.

Lemma 2.9 ([14]). Let $\mathrm{X}$ be a nonempty set and $\mathrm{g}$ a self-mapping on $\mathrm{X}$. Then there exists a subset $\mathrm{E} \subseteq \mathrm{X}$ such that $\mathrm{g}(\mathrm{E})=\mathrm{g}(\mathrm{X})$ and $\mathrm{g}: \mathrm{E} \rightarrow \mathrm{X}$ is one-one.

Lemma 2.10 ([4]). Let $\mathrm{f}$ and $\mathrm{g}$ be two self-mappings defined on a nonempty set $\mathrm{X}$. If $\mathrm{f}$ and $\mathrm{g}$ are weakly compatible, then every point of coincidence of $\mathrm{f}$ and $\mathrm{g}$ is also a coincidence point of $\mathrm{f}$ and $\mathrm{g}$.

\section{3. $\prec \succ$-theoretic notions}

In this section, we recall and define $\prec \succ$-variants of several set-theoretic, metrical and order-relational notions.

Definition 3.1 ([2]). Let $(X, \preceq)$ be an ordered set and $f$ and $g$ two self-mappings on $X$. We say that $f$ is 
g-comparable (or weakly g-monotone or $(g, \prec \succ)$-preserving), if for any $x, y \in X$,

$$
g(x) \prec \succ g(y) \Rightarrow f(x) \prec \succ f(y) .
$$

Notice that if we set $g=I$, the identity mapping on $X$ in Definition 3.1, then $f$ is called comparable (or weakly monotone or $\prec \succ$-preserving) mapping. Clearly every g-monotone mapping is $g$-comparable.

Recently Alam and Imdad [3] formulated the variants of bounded sequences and monotone sequences with respect to relation $\prec \succ$ by introducing the following.

Definition $3.2([3])$. Let $(X, \preceq)$ be an ordered set and $\left\{x_{n}\right\}$ a sequence in $X$. Then

(i) $\left\{x_{n}\right\}$ is said to be termwise bounded, if there is an element $z \in X$ such that each term of $\left\{x_{n}\right\}$ is comparable with $z$, i.e.,

$$
x_{\mathrm{n}} \prec \succ z, \quad \forall \mathrm{n} \in \mathbb{N}_{0},
$$

so that $z$ is a c-bound of $\left\{x_{n}\right\}$, and

(ii) $\left\{x_{n}\right\}$ is said to termwise monotone (or $\prec \succ$-preserving), if consecutive terms of $\left\{x_{n}\right\}$ are comparable, i.e.,

$$
x_{\mathrm{n}} \prec \succ x_{\mathrm{n}+1}, \quad \forall \mathrm{n} \in \mathbb{N}_{0} .
$$

Clearly all bounded above as well as bounded below sequences are termwise bounded and all monotone sequences are termwise monotone.

Definition $3.3([3])$. An ordered set $(X, \preceq)$ is called sequentially chainable, if range of every termwise monotone sequence in $X$ remains a totally ordered subset of $X$.

Proposition 3.4 ([3]). The following are equivalent:

(i) $(X, \preceq)$ is sequentially chainable;

(ii) $\prec \succ$ is transitive on range of every termwise monotone sequence in $\mathrm{X}$;

(iii) for every termwise monotone sequence $\left\{x_{n}\right\}$ in $X$,

$$
x_{\mathrm{n}} \prec \succ x_{\mathrm{m}}, \quad \forall \mathrm{n}, \mathrm{m} \in \mathbb{N}_{0} .
$$

Let $(X, d, \preceq)$ be an ordered metric space and $\left\{x_{n}\right\}$ a sequence in $X$. If $\left\{x_{n}\right\}$ is termwise monotone and $x_{n} \stackrel{d}{\longrightarrow} x$, then we denote it symbolically by $x_{n} \uparrow x$.

Alam and Imdad [3] formulated the following notion by using a suitable property on ordered metric space (in order to avoid the necessity of continuity requirement in Ran-Reurings Theorem) utilized by Nieto and Rodríguez-López [27].

Definition 3.5 ([3]). Let $(X, d, \preceq)$ be an ordered metric space. We say that $(X, d, \preceq)$ has TCC (termwise monotone-convergence-c-bound) property, if every termwise monotone convergent sequence $\left\{x_{n}\right\}$ in $X$ has a subsequence, which is termwise bounded by the limit (of the sequence) as a c-bound, i.e.,

$$
x_{\mathrm{n}} \uparrow x \Rightarrow \text { there exists a subsequence }\left\{x_{n_{k}}\right\} \text { of }\left\{x_{n}\right\} \text { with } x_{n_{k}} \prec \succ x, \quad \forall k \in \mathbb{N}_{0} .
$$

Definition $3.6([3])$. Let $(X, d, \preceq)$ be an ordered metric space and $g$ a self-mapping on $X$. We say that $(X, d, \preceq)$ has $g$-TCC property, if every termwise monotone convergent sequence $\left\{x_{n}\right\}$ in $X$ has a subsequence, whose g-image is termwise bounded by g-image of limit (of the sequence) as a c-bound, i.e.,

$$
x_{n} \uparrow x \Rightarrow \text { there exists a subsequence }\left\{x_{n_{k}}\right\} \text { of }\left\{x_{n}\right\} \text { with } g\left(x_{n_{k}}\right) \prec \succ g(x), \quad \forall k \in \mathbb{N}_{0} .
$$
3.5 .

Notice that under the restriction $\mathrm{g}=\mathrm{I}$, the identity mapping on $\mathrm{X}$, Definition 3.6 reduces to Definition Inspired by Jleli et al. [18], Alam and Imdad [2] defined the following notion. 
Definition 3.7 ([2]). Let $(X, \preceq)$ be an ordered set and $f$ and $g$ two self-mappings on $X$. We say that $(X, \preceq)$ is $(f, g)$-directed, if for each pair $x, y \in X$, there exists a $z \in X$ such that $f(x) \prec \succ g(z)$ and $f(y) \prec \succ g(z)$.

In cases $g=I$ and $f=g=I$ (where I denotes the identity mapping on $X$ ), $(X, \preceq)$ is called $f$-directed and directed respectively.

Inspired by Turinici [34, 35], Alam and Imdad [2] defined the following notion.

Definition 3.8 ([2]). Let $(X, \preceq)$ be an ordered set, $E \subseteq X$ and $a, b \in E$. A finite subset $\left\{e_{1}, e_{2}, \ldots, e_{k}\right\}$ of $E$ is called $\prec \succ$-chain between $a$ and $b$ in $E$, if

(i) $k \geqslant 2$;

(ii) $e_{1}=a$ and $e_{k}=b$;

(iii) $e_{i} \prec \succ e_{i+1}$ for each $i(1 \leqslant i \leqslant k-1)$.

In the following lines, we adopt several well-known metrical notions such as: completeness, closedness, continuity, g-continuity and compatibility with respect to relation $\prec \succ$.

Definition 3.9. An ordered metric space $(X, d, \preceq)$ is called $\prec \succ$-complete, if every termwise monotone Cauchy sequence in $X$ converges.

Remark 3.10. If $(X, d)$ is a complete metric space, then for each partial order $\preceq$ defined on $X$, the ordered metric space $(X, d, \preceq)$ is $\prec \succ$-complete.

Definition 3.11. Let $(X, d, \preceq)$ be an ordered metric space. A subset $E$ of $X$ is called $\prec \succ$-closed if for any sequence $\left\{x_{n}\right\} \subset E$,

$$
x_{\mathrm{n}} \uparrow x \Rightarrow x \in \mathrm{E} .
$$

Remark 3.12. Every closed subset of an ordered metric space is $\prec \succ$-closed.

Proposition 3.13. $A \prec \succ$-complete subspace of an ordered metric space is $\prec \succ$-closed.

Proof. Let $(X, d, \preceq)$ be an ordered metric space. Suppose that $Y$ is $\prec \succ$-complete subspace of $X$. Take a sequence $\left\{x_{n}\right\} \subset Y$ such that $x_{n} \uparrow x \in X$. As each convergent sequence is Cauchy, $\left\{x_{n}\right\}$ is a termwise monotone Cauchy sequence in $Y$. Hence, $\prec \succ$-completeness of $Y$ implies that the limit of $\left\{x_{n}\right\}$ must lie in $Y$, i.e., $x \in Y$. Therefore, $Y$ is $\prec \succ$-closed.

Proposition 3.14. $A \prec \succ$-closed subspace of $a \prec \succ$-complete ordered metric space is $\prec \succ$-complete.

Proof. Let $(X, d, \preceq)$ be a $\prec \succ$-complete metric space. Suppose that $Y$ is $\prec \succ$-closed subspace of $X$. Take a termwise monotone Cauchy sequence $\left\{x_{n}\right\}$ in $Y$. As $X$ is $\prec \succ$-complete, there exists a $x \in X$ such that $x_{n} \stackrel{d}{\longrightarrow} x$ and so $x_{n} \downarrow x$. Hence, $\prec \succ$-closeness of $Y$ implies that $x \in Y$. Therefore, $Y$ is $\prec \succ$-complete.

Definition 3.15. Let $(X, d, \preceq)$ be an ordered metric space, $f$ a self-mapping on $X$ and $x \in X$. We say that $f$ is $\prec \succ$-continuous at $x$, if for any sequence $\left\{x_{n}\right\} \subset X$,

$$
x_{\mathrm{n}} \mathfrak{\imath} x \Rightarrow \mathrm{f}\left(x_{\mathrm{n}}\right) \stackrel{\mathrm{d}}{\longrightarrow} \mathrm{f}(\mathrm{x}) .
$$

Moreover, $f$ is called $\prec \succ$-continuous, if it is $\prec \succ$-continuous at each point of $X$.

Remark 3.16. Every continuous mapping defined on an ordered metric space is $\prec \succ$-continuous.

Definition 3.17. Let $(X, d, \preceq)$ be an ordered metric space, $f$ and $g$ two self-mappings on $X$ and $x \in X$. We say that $f$ is $(g, \prec \succ)$-continuous at $x$, if for any sequence $\left\{x_{n}\right\} \subset X$,

$$
g\left(x_{n}\right) \downarrow g(x) \Rightarrow f\left(x_{n}\right) \stackrel{d}{\longrightarrow} f(x) .
$$

Moreover, $f$ is called $(g, \prec \succ)$-continuous, if it is $(g, \prec \succ)$-continuous at each point of $X$. 
Notice that particularly, at $\mathrm{g}=\mathrm{I}$, the identity mapping on $\mathrm{X}$, Definition 3.17 reduces to Definition 3.15. Remark 3.18. Every g-continuous mapping defined on an ordered metric space is $(g, \prec \succ)$-continuous.

Definition 3.19. Let $(X, d, \preceq)$ be an ordered metric space and $f$ and $g$ two self-mappings on $X$. We say that $f$ and $g$ are $\prec \succ$-compatible, if for any sequence $\left\{x_{n}\right\} \subset X$ and for any $z \in X$,

$$
g\left(x_{n}\right) \uparrow z \text { and } f\left(x_{n}\right) \uparrow z \Rightarrow \lim _{n \rightarrow \infty} d\left(g f x_{n}, f g x_{n}\right)=0 .
$$

Remark 3.20. In an ordered metric space, commutativity $\Rightarrow$ weak commutativity $\Rightarrow$ compatibility $\Rightarrow \prec \succ$ compatibility $\Rightarrow$ weak compatibility.

Finally, we state the recent coincidence theorem in ordered metric spaces for linear contractivity conditions without involving g-monotone mappings proved by Alam and Imdad [2].

Theorem 3.21 ([2]). Let $(\mathrm{X}, \mathrm{d}, \preceq)$ be an ordered metric space and $\mathrm{f}$ and $\mathrm{g}$ two self-mappings on $\mathrm{X}$. Suppose that the following conditions hold:

(a) $f(X) \subseteq g(X)$;

(b) $\mathrm{f}$ is g-comparable;

(c) there exists $x_{0} \in X$ such that $\mathrm{g}\left(\mathrm{x}_{0}\right) \prec \succ \mathrm{f}\left(\mathrm{x}_{0}\right)$;

(d) there exists $\alpha \in[0,1)$ such that

$$
d(f x, f y) \leqslant \alpha d(g x, g y), \quad \forall x, y \in X
$$

with $\mathrm{g}(\mathrm{x}) \prec \succ \mathrm{g}(\mathrm{y})$;

(e) (e1) $(\mathrm{X}, \mathrm{d})$ is complete;

(e2) f and $g$ are compatible;

(e3) $g$ is continuous;

(e4) either $\mathrm{f}$ is continuous or $(\mathrm{X}, \mathrm{d}, \preceq)$ has $g$-TCC property;

or

$\left(\mathrm{e}^{\prime}\right)\left(\mathrm{e}^{\prime} 1\right)$ either $(\mathrm{fX}, \mathrm{d})$ or $(\mathrm{gX}, \mathrm{d})$ is complete;

$\left(\mathrm{e}^{\prime 2}\right)$ either $\mathrm{f}$ is $\mathrm{g}$-continuous or $\mathrm{f}$ and $\mathrm{g}$ are continuous or $(\mathrm{gX}, \mathrm{d}, \preceq)$ has TCC property.

Then $\mathrm{f}$ and $\mathrm{g}$ have a coincidence point.

\section{Results on coincidence points}

We are equipped to prove the following result, which guarantees the existence of a coincidence point under $\varphi$-contractivity condition.

Theorem 4.1. Let $(\mathrm{X}, \mathrm{d}, \preceq)$ be an ordered metric space and $\mathrm{f}$ and $\mathrm{g}$ two self-mappings on $\mathrm{X}$. Let $\mathrm{Y}$ be $a \prec \succ$-complete subspace of $X$ such that $(Y, \preceq)$ is sequentially chainable. Suppose that the following conditions hold:

(a) $f(X) \subseteq g(X) \cap Y$;

(b) $\mathrm{f}$ is g-comparable and $\mathrm{g}$-admissible;

(c) there exists $x_{0} \in X$ such that $\mathrm{g}\left(\mathrm{x}_{0}\right) \prec \succ \mathrm{f}\left(\mathrm{x}_{0}\right)$;

(d) there exists $\varphi \in \Omega$ such that

$$
d(f x, f y) \leqslant \varphi(d(g x, g y)), \quad \forall x, y \in X
$$

with $\mathrm{g}(\mathrm{x}) \prec \succ \mathrm{g}(\mathrm{y})$; 
(e) (e1) f and g are $\prec \succ$-compatible;

(e2) $\mathrm{g}$ is $\prec \succ$-continuous;

(e3) either $\mathrm{f}$ is $\prec \succ$-continuous or $(\mathrm{Y}, \mathrm{d}, \preceq)$ has g-TCC property;

or alternately

$\left(\mathrm{e}^{\prime}\right)\left(\mathrm{e}^{\prime} 1\right) \mathrm{Y} \subseteq \mathrm{g}(\mathrm{X})$;

$\left(\mathrm{e}^{\prime 2}\right)$ either $\mathrm{f}$ is $(\mathrm{g}, \prec \succ)$-continuous or $\mathrm{f}$ and $\mathrm{g}$ are continuous or $(\mathrm{Y}, \mathrm{d}, \preceq)$ has TCC property.

Then $\mathrm{f}$ and $\mathrm{g}$ have a coincidence point.

Proof. Firstly, we notice that the assumption (a) is equivalent to say that $f(X) \subseteq g(X)$ and $f(X) \subseteq Y$. Now, in view of assumption (c), if $g\left(x_{0}\right)=f\left(x_{0}\right)$, then $x_{0}$ is a coincidence point of $f$ and $g$ and hence proof is completed. Otherwise, using assumption $f(X) \subseteq g(X)$, we can choose $x_{1} \in X$ such that $g\left(x_{1}\right)=f\left(x_{0}\right)$. Again from $f(X) \subseteq g(X)$, we can choose $x_{2} \in X$ such that $g\left(x_{2}\right)=f\left(x_{1}\right)$. Continuing this process, we define a sequence $\left\{x_{n}\right\} \subset X$ of joint iterates such that

$$
\mathrm{g}\left(x_{\mathrm{n}+1}\right)=\mathrm{f}\left(x_{\mathrm{n}}\right), \quad \forall \mathrm{n} \in \mathbb{N}_{0} .
$$

Now, we claim that $\left\{g x_{n}\right\}$ is a termwise monotone sequence, i.e.,

$$
\mathrm{g}\left(\mathrm{x}_{\mathrm{n}}\right) \prec \succ \mathrm{g}\left(\mathrm{x}_{\mathrm{n}+1}\right), \quad \forall \mathrm{n} \in \mathbb{N}_{0} .
$$

We prove this fact by mathematical induction. On using (4.1) with $n=0$ and assumption (c), we have

$$
g\left(x_{0}\right) \prec \succ f\left(x_{0}\right)=g\left(x_{1}\right) \text {. }
$$

Thus, (4.2) holds for $n=0$. Suppose that (4.2) holds for $n=r>0$, i.e.,

$$
g\left(x_{r}\right) \prec \succ g\left(x_{r+1}\right),
$$

which on using (4.1) and g-comparability of $f$ gives rise

$$
g\left(x_{r+1}\right)=f\left(x_{r}\right) \prec \succ f\left(x_{r+1}\right)=g\left(x_{r+2}\right),
$$

i.e., (4.2) holds for $n=r+1$. Hence, by induction, (4.2) holds for all $n \in \mathbb{N}_{0}$.

In view of (4.1) and (4.2), the sequence $\left\{f x_{n}\right\}$ is also a termwise monotone sequence, i.e.,

$$
\mathrm{f}\left(\mathrm{x}_{\mathrm{n}}\right) \prec \succ \mathrm{f}\left(\mathrm{x}_{\mathrm{n}+1}\right), \quad \forall \mathrm{n} \in \mathbb{N}_{0} .
$$

If $g\left(x_{n_{0}}\right)=g\left(x_{n_{0}+1}\right)$ for some $n_{0} \in \mathbb{N}$, then using (4.1), we have $g\left(x_{n_{0}}\right)=f\left(x_{n_{0}}\right)$, i.e., $x_{n_{0}}$ is a coincidence point of $f$ and $g$ so that we are through. On the other hand, if $g\left(x_{n}\right) \neq g\left(x_{n+1}\right)$ for each $\mathrm{n} \in \mathbb{N}_{0}$, we can define a sequence $\left\{\mathrm{d}_{\mathfrak{n}}\right\}_{\mathfrak{n}=0}^{\infty} \subset(0, \infty)$, where

$$
d_{n}:=d\left(g x_{n}, g x_{n+1}\right) .
$$

On using (4.1), (4.2), (4.4) and assumption (d), we obtain

$$
\begin{aligned}
d_{n+1} & =d\left(g x_{n+1}, g x_{n+2}\right) \\
& =d\left(f x_{n}, f x_{n+1}\right) \\
& \leqslant \varphi\left(d\left(g x_{n}, g x_{n+1}\right)\right) \\
& =\varphi\left(d_{n}\right),
\end{aligned}
$$

so that

$$
d_{n+1} \leqslant \varphi\left(d_{n}\right)
$$

Hence by Lemma 2.7, we obtain

$$
\lim _{n \rightarrow \infty} d_{n}=\lim _{n \rightarrow \infty} d\left(g x_{n}, g x_{n+1}\right)=0 .
$$

Next, we show that $\left\{g x_{n}\right\}$ is a Cauchy sequence. On contrary suppose that $\left\{g x_{n}\right\}$ is not a Cauchy, then 
owing to Lemma 2.8, there exist $\epsilon>0$ and two subsequences $\left\{g x_{n_{k}}\right\}$ and $\left\{g x_{m_{k}}\right\}$ of $\left\{g x_{n}\right\}$ such that $k \leqslant m_{k}<n_{k}$ and $d\left(g x_{m_{k}}, g x_{n_{k}}\right)>\epsilon \geqslant d\left(g x_{m_{k}}, g x_{n_{k-1}}\right)$ for all $k \in \mathbb{N}$. Further, in view of (4.5), Lemma 2.8 assures us that

$$
\lim _{k \rightarrow \infty} d\left(g x_{m_{k}}, g x_{n_{k}}\right)=\lim _{k \rightarrow \infty} d\left(g x_{m_{k}+1}, g x_{n_{k}+1}\right)=\epsilon .
$$

Denote $r_{k}:=d\left(g x_{m_{k}}, g x_{n_{k}}\right)$. Owing to (4.1), we have $\left\{g x_{n}\right\} \subset f(X) \subseteq Y$ so that $\left\{g x_{n}\right\}$ is termwise monotone sequence in $Y$ (due to $(4.2)$ ). As $(Y, \preceq)$ is sequentially chainable, by using Proposition 3.4 , we obtain $g\left(x_{m_{k}}\right) \prec \succ g\left(x_{n_{k}}\right)$. On using (4.1) and assumption (d), we obtain

$$
\begin{aligned}
\mathrm{d}\left(g x_{m_{k}+1}, g x_{n_{k}+1}\right) & =d\left(f x_{m_{k}}, f x_{n_{k}}\right) \\
& \leqslant \varphi\left(d\left(g x_{m_{k}}, g x_{n_{k}}\right)\right) \\
& =\varphi\left(r_{k}\right),
\end{aligned}
$$

so that

$$
\mathrm{d}\left(\mathrm{g} \mathrm{x}_{\mathrm{m}_{\mathrm{k}}+1}, \mathrm{~g} \mathrm{x}_{\mathrm{n}_{\mathrm{k}}+1}\right) \leqslant \varphi\left(\mathrm{r}_{\mathrm{k}}\right) .
$$

On taking limit superior as $k \longrightarrow \infty$ in (4.7) and using (4.6) and the definition of $\Omega$, we have

$$
\epsilon=\limsup _{k \rightarrow \infty} d\left(g x_{m_{k}+1}, g x_{n_{k}+1}\right) \leqslant \limsup _{k \rightarrow \infty} \varphi\left(r_{k}\right)=\limsup _{r_{k} \rightarrow \epsilon^{+}} \varphi\left(r_{k}\right)<\epsilon,
$$

which is a contradiction yielding thereby $\left\{g x_{n}\right\}$ is a Cauchy sequence.

Therefore $\left\{g x_{\mathfrak{n}}\right\}$ is a termwise monotone Cauchy sequence in $Y$. As $Y$ is $\prec \succ$-complete, there exists $z \in Y$ such that $\lim _{n \rightarrow \infty} g\left(x_{n}\right)=z$, which combining with (4.2), gives rise

$$
g\left(x_{n}\right) \downarrow z .
$$

On using (4.1), (4.3) and (4.8), we obtain

$$
f\left(x_{n}\right) \uparrow z .
$$

Now, we use assumptions (e) and ( $\left.\mathrm{e}^{\prime}\right)$ to accomplish the proof. Assume that (e) holds. Using assumption (e2) (i.e., $\prec \succ$-continuity of $g$ ) in (4.8) and (4.9), we have

$$
\begin{aligned}
& \lim _{n \rightarrow \infty} g\left(g x_{n}\right)=g(z), \\
& \lim _{n \rightarrow \infty} g\left(f x_{n}\right)=g(z) .
\end{aligned}
$$

On using (4.8), (4.9) and assumption (e1) (i.e., $\prec \succ$-compatibility of $f$ and g), we obtain

$$
\lim _{n \rightarrow \infty} d\left(g f x_{n}, f g x_{n}\right)=0 .
$$

Now, we show that $z$ is a coincidence point of $f$ and $g$. To accomplish this, we use assumption (e3). Suppose that $f$ is $\prec \succ$-continuous. On using (4.8) and $\prec \succ$-continuity of $f$, we obtain

$$
\lim _{n \rightarrow \infty} f\left(g x_{n}\right)=f(z)
$$

On using (4.11), (4.12), (4.13) and continuity of $d$, we obtain

$$
\begin{aligned}
d(g z, f z) & =d\left(\lim _{n \rightarrow \infty} g f x_{n}, \lim _{n \rightarrow \infty} f g x_{n}\right) \\
& =\lim _{n \rightarrow \infty} d\left(g f x_{n}, f g x_{n}\right) \\
& =0,
\end{aligned}
$$

so that

$$
g(z)=f(z) .
$$


Thus $z \in X$ is a coincidence point of $f$ and $g$ and hence we are through. Alternately, suppose that $(Y, d, \preceq)$ has $g$-TCC property, then due to availability of (4.8), there exists a subsequence $\left\{g x_{n_{k}}\right\}$ of $\left\{g x_{n}\right\}$ such that

$$
g\left(g x_{n_{k}}\right) \prec \succ g(z), \quad \forall k \in \mathbb{N}_{0} .
$$

On using (4.14) and assumption (d), we obtain

$$
\mathrm{d}\left(f g x_{n_{k}}, f z\right) \leqslant \varphi\left(d\left(g g x_{n_{k}}, g z\right)\right), \quad \forall k \in \mathbb{N}_{0} .
$$

Now, we asserts that

$$
\mathrm{d}\left(\mathrm{fg} \mathrm{x}_{\mathrm{n}_{\mathrm{k}}}, \mathrm{fz}\right) \leqslant \mathrm{d}\left(\mathrm{ggx_{n_{ \textrm {k } } }}, \mathrm{gz}\right), \quad \forall \mathrm{k} \in \mathbb{N} .
$$

On account of two different possibilities arising here, we consider a partition $\left\{\mathbb{N}^{0}, \mathbb{N}^{+}\right\}$of $\mathbb{N}$, i.e., $\mathbb{N}^{0} \cup$ $\mathbb{N}^{+}=\mathbb{N}$ and $\mathbb{N}^{0} \cap \mathbb{N}^{+}=\emptyset$ verifying that

(i) $\mathrm{d}\left(g g x_{n_{k}}, g z\right)=0, \forall k \in \mathbb{N}^{0}$;

(ii) $\mathrm{d}\left(g g x_{n_{k}}, g z\right)>0, \forall k \in \mathbb{N}^{+}$.

In case (i), on using g-admissibility of $f$, we get $d\left(f g x_{n_{k}}, f z\right)=0$, for all $k \in \mathbb{N}^{0}$ and hence (4.15) holds for all $k \in \mathbb{N}^{0}$. In case (ii), owing to the definition of $\Omega$, we have $d\left(f g x_{n_{k}}, f z\right) \leqslant \varphi\left(d\left(g g x_{n_{k}}, g z\right)\right)<$ $\mathrm{d}\left(\mathrm{ggx}_{\mathrm{n}_{k}}, \mathrm{gz}\right)$, for all $k \in \mathbb{N}^{+}$and hence (4.15) holds for all $k \in \mathbb{N}^{+}$. Thus (4.15) holds for all $k \in \mathbb{N}$. On using triangular inequality, (4.10), (4.11), (4.12) and (4.15), we get

$$
\begin{aligned}
d(g z, f z) & \leqslant d\left(g z, g f x_{n_{k}}\right)+d\left(g f x_{n_{k}}, f g x_{n_{k}}\right)+d\left(f g x_{n_{k}}, f z\right) \\
& \leqslant d\left(g z, g f x_{n_{k}}\right)+d\left(g f x_{n_{k}}, f g x_{n_{k}}\right)+d\left(g g x_{n_{k}}, g z\right) \\
& \rightarrow 0 \text { as } k \rightarrow \infty,
\end{aligned}
$$

so that

$$
g(z)=f(z)
$$

Thus $z \in X$ is a coincidence point of $f$ and $g$.

Now, assume that $\left(e^{\prime}\right)$ holds. Owing to assumption ( $\left.e^{\prime} 1\right)$ (i.e., $Y \subseteq g(X)$ ), we can find some $u \in X$ such that $z=g(u)$. Hence, (4.8) and (4.9) respectively reduce to

$$
\begin{aligned}
& g\left(x_{n}\right) \uparrow g(u), \\
& f\left(x_{n}\right) \downarrow g(u) .
\end{aligned}
$$

Now, we show that $u$ is a coincidence point of $f$ and $g$. To accomplish this, we use assumption $\left(e^{\prime} 2\right)$. Firstly, suppose that $f$ is $(g, \prec \succ)$-continuous, then using (4.16), we get

$$
\lim _{n \rightarrow \infty} f\left(x_{n}\right)=f(u) .
$$

On using (4.17) and (4.18), we get

$$
g(u)=f(u)
$$

Hence, we are done. Secondly, suppose that $f$ and $g$ are continuous. Owing to Lemma 2.9, there exists a subset $E \subseteq X$ such that $g(E)=g(X)$ and $g: E \rightarrow X$ is one-one. Now, define $T: g(E) \rightarrow g(X)$ by

$$
T(g a)=f(a), \quad \forall g(a) \in g(E), \quad \text { where } a \in E .
$$

As $g: E \rightarrow X$ is one-one and $f(X) \subseteq g(X), T$ is well-defined. Again since $f$ and $g$ are continuous, it follows that $T$ is continuous. Using $g(X)=g(E)$, assumptions (a) and $\left(e^{\prime} 1\right)$ reduce to respectively $f(X) \subseteq g(E) \cap Y$ 
and $Y \subseteq g(E)$, which follows that, without loss of generality, we are able to construct $\left\{x_{n}\right\}_{\mathfrak{n}=1}^{\infty} \subset E$ satisfying (4.1) and to choose $u \in E$. On using (4.16), (4.17), (4.19) and continuity of $T$, we get

$$
f(u)=T(g u)=T\left(\lim _{n \rightarrow \infty} g x_{n}\right)=\lim _{n \rightarrow \infty} T\left(g x_{n}\right)=\lim _{n \rightarrow \infty} f\left(x_{n}\right)=g(u) .
$$

Thus $u \in X$ is a coincidence point of $f$ and $g$ and hence we are through. Finally, suppose that $(Y, d, \preceq)$ has TCC property, then due to availability of (4.16), there exists a subsequence $\left\{g x_{n_{k}}\right\}$ of $\left\{g x_{n}\right\}$ such that

$$
\mathrm{g}\left(\mathrm{x}_{\mathrm{n}_{\mathrm{k}}}\right) \prec \succ \mathrm{g}(\mathrm{u}), \quad \forall \mathrm{k} \in \mathbb{N}_{0} .
$$

On using (4.1), (4.20) and assumption (d), we obtain

$$
d\left(g x_{n_{k}+1}, f u\right)=d\left(f x_{n_{k}}, f u\right) \leqslant \varphi\left(d\left(g x_{n_{k}}, g u\right)\right), \quad \forall k \in \mathbb{N}_{0} .
$$

We asserts that

$$
\mathrm{d}\left(\mathrm{g} \mathrm{x}_{\mathrm{n}_{\mathrm{k}}+1}, \mathrm{fu}\right) \leqslant \mathrm{d}\left(\mathrm{g} \mathrm{x}_{\mathrm{n}_{\mathrm{k}}}, \mathrm{gu}\right), \quad \forall \mathrm{k} \in \mathbb{N} .
$$

On account of two different possibilities arising here, we consider a partition $\left\{\mathbb{N}^{0}, \mathbb{N}^{+}\right\}$of $\mathbb{N}$, i.e., $\mathbb{N}^{0} \cup$ $\mathbb{N}^{+}=\mathbb{N}$ and $\mathbb{N}^{0} \cap \mathbb{N}^{+}=\emptyset$ verifying that

(i) $\mathrm{d}\left(g \mathrm{gx}_{\mathrm{n}_{\mathrm{k}}}, \mathrm{gu}\right)=0 \quad \forall \mathrm{k} \in \mathbb{N}^{0}$;

(ii) $\mathrm{d}\left(\mathrm{gx_{n_{k } }}, \mathrm{gu}\right)>0 \forall \mathrm{k} \in \mathbb{N}^{+}$.

In case (i), on using $g$-admissibility of $f$, we get $d\left(f x_{n_{k}}, f u\right)=0$, for all $k \in \mathbb{N}^{0}$, which in view of (4.1), gives rise $d\left(g x_{n_{k}+1}, f u\right)=0$, for all $k \in \mathbb{N}^{0}$ and hence (4.21) holds for all $k \in \mathbb{N}^{0}$. In case (ii), by the definition of $\Omega$, we have $d\left(g x_{n_{k}+1}, f u\right) \leqslant \varphi\left(d\left(g x_{n_{k}}, g u\right)\right)<d\left(g x_{n_{k}}, g u\right)$, for all $k \in \mathbb{N}^{+}$and hence (4.21) holds for all $n \in \mathbb{N}^{+}$. Thus (4.21) holds for all $k \in \mathbb{N}$. On using (4.16), (4.21) and continuity of $d$, we get

$$
\begin{aligned}
d(g u, f u) & =d\left(\lim _{n \rightarrow \infty} g x_{n+1}, f u\right) \\
& =\lim _{k \rightarrow \infty} d\left(g x_{n_{k}+1}, f u\right) \\
& \leqslant \lim _{k \rightarrow \infty} d\left(g x_{n_{k}}, g u\right) \\
& =0
\end{aligned}
$$

so that

$$
g(u)=f(u) \text {. }
$$

Hence, $u$ is a coincidence point of $f$ and $g$. This completes the proof.

Remark 4.2. In view of Proposition 1.1, Theorem 4.1 remains true, if we replace the class $\Omega$ by anyone of the classes $\Psi, \Theta$, I and $\Phi$.

With a view to deduce a natural consequence, we particularize Theorem 4.1 by assuming the $\prec \succ$ completeness of whole space $X$.

Corollary 4.3. Let $(\mathrm{X}, \mathrm{d}, \preceq)$ be $a \prec \succ$-complete ordered metric space such that $(\mathrm{X}, \preceq)$ is sequentially chainable and $\mathrm{f}$ and $\mathrm{g}$ two self-mappings on $\mathrm{X}$. Suppose that the following conditions hold:

(a) $f(X) \subseteq g(X)$;

(b) $\mathrm{f}$ is g-comparable and $\mathrm{g}$-admissible;

(c) there exists $x_{0} \in X$ such that $\mathrm{g}\left(\mathrm{x}_{0}\right) \prec \succ \mathrm{f}\left(\mathrm{x}_{0}\right)$;

(d) there exists $\varphi \in \Omega$ such that

$$
\mathrm{d}(\mathrm{fx}, \mathrm{fy}) \leqslant \varphi(\mathrm{d}(\mathrm{gx}, \mathrm{gy})), \quad \forall x, y \in X
$$

with $\mathrm{g}(\mathrm{x}) \prec \succ \mathrm{g}(\mathrm{y})$; 
(e) (e1) f and g are $\prec \succ$-compatible;

(e2) g is $\prec \succ$-continuous;

(e3) either $\mathrm{f}$ is $\prec \succ$-continuous or $(\mathrm{X}, \mathrm{d}, \preceq)$ has g-TCC property;

or alternately

$\left(\mathrm{e}^{\prime}\right)\left(\mathrm{e}^{\prime} 1\right)$ there exists $a \prec \succ$-closed subspace $\mathrm{Y}$ of $\mathrm{X}$ such that $\mathrm{f}(\mathrm{X}) \subseteq \mathrm{Y} \subseteq \mathrm{g}(\mathrm{X})$;

$\left(\mathrm{e}^{\prime} 2\right)$ either $\mathrm{f}$ is $(\mathrm{g}, \prec \succ)$-continuous or $\mathrm{f}$ and $\mathrm{g}$ are continuous or $(\mathrm{Y}, \mathrm{d}, \preceq)$ has TCC property.

Then $\mathrm{f}$ and $\mathrm{g}$ have a coincidence point.

Proof. The result corresponding to part (e) follows easily on setting $Y=X$ in Theorem 4.1, while the same (result) in the presence of part ( $\left.\mathrm{e}^{\prime}\right)$ follows using Proposition 3.14.

Corollary 4.4. Theorem 4.1 (also Corollary 4.3) remains true, if we replace condition " $\mathrm{f}$ is $\mathrm{g}$-admissible" by one of the following conditions (besides retaining the rest of the hypotheses):

(i) $\varphi(0)=0$;

(ii) $\mathrm{g}$ is one-one.

Proof. Suppose that (i) holds. Take $x, y \in X$ such that $g(x)=g(y)$, then $g(x) \preceq g(y)$ and $g(x) \succeq g(y)$. On applying these points to the contractivity condition (d), we get

$$
d(f x, f y) \leqslant \varphi(d(g x, g y))=\varphi(0)=0,
$$

which implies that $f(x)=f(y)$. It follows that $f$ is $g$-admissible.

Suppose that (ii) holds. Take $x, y \in X$ such that $g(x)=g(y)$. As $g$ is one-one, we get $x=y$, which implies that $f(x)=f(y)$. Hence, $f$ is g-admissible.

Using the fact that g-monotonicity implies g-comparability and Proposition 2.6, the following consequence of Theorem 4.1 and Corollary 4.3 trivially holds:

Corollary 4.5. Theorem 4.1 (also Corollary 4.3) remains true, if we replace condition (b) by the following condition (besides retaining the rest of the hypotheses):

$(\mathrm{b})^{\prime} \mathrm{f}$ is g-monotone.

Remark 4.6. Notice that Corollary 4.5 improves the main result of Alam and Imdad [3].

Now, we prove the corresponding result of Theorem 4.1 under linear contractivity condition, as follows.

Theorem 4.7. Let $(\mathrm{X}, \mathrm{d}, \preceq)$ be an ordered metric space and $\mathrm{f}$ and $\mathrm{g}$ two self-mappings on $\mathrm{X}$. Let $\mathrm{Y}$ be $a \prec \succ$-complete subspace of $\mathrm{X}$. Suppose that the following conditions hold:

(a) $f(X) \subseteq g(X) \cap Y$;

(b) $\mathrm{f}$ is g-comparable;

(c) there exists $x_{0} \in X$ such that $\mathrm{g}\left(\mathrm{x}_{0}\right) \prec \succ \mathrm{f}\left(\mathrm{x}_{0}\right)$;

(d) there exists $\alpha \in[0,1)$ such that

$$
d(f x, f y) \leqslant \alpha d(g x, g y), \quad \forall x, y \in X
$$

with $\mathrm{g}(\mathrm{x}) \prec \succ \mathrm{g}(\mathrm{y})$; 
(e) (e1) f and g are $\prec \succ$-compatible;

(e2) g is $\prec \succ$-continuous;

(e3) either $\mathrm{f}$ is $\prec \succ$-continuous or $(\mathrm{Y}, \mathrm{d}, \preceq)$ has g-TCC property;

or alternately

$\left(\mathrm{e}^{\prime}\right)\left(\mathrm{e}^{\prime} 1\right) \mathrm{Y} \subseteq \mathrm{g}(\mathrm{X})$;

$\left(\mathrm{e}^{\prime 2}\right)$ either $\mathrm{f}$ is $(\mathrm{g}, \prec \succ)$-continuous or $\mathrm{f}$ and $\mathrm{g}$ are continuous or $(\mathrm{Y}, \mathrm{d}, \preceq)$ has TCC property.

Then $\mathrm{f}$ and $\mathrm{g}$ have a coincidence point.

Proof. This result follows from Theorem 4.1 on setting $\varphi(t)=\alpha t$ with $\alpha \in[0,1)$ besides removing the following assumptions:

(i) $(Y, \preceq)$ is sequentially chainable;

(ii) $f$ is $g$-admissible.

Condition (i) is used to prove that $\left\{g x_{n}\right\}$ is a Cauchy sequence. In this case, using the analogous technique as utilized in Theorem 4.1, we obtain

$$
d\left(g x_{n+1}, g x_{n+2}\right)=d\left(f x_{n}, f x_{n+1}\right) \leqslant \alpha d\left(g x_{n}, g x_{n+1}\right), \quad \forall n \in \mathbb{N}_{0},
$$

so that

$$
\mathrm{d}\left(\mathrm{g} \mathrm{x}_{\mathrm{n}}, \mathrm{g} \mathrm{x}_{\mathrm{n}+1}\right) \leqslant \alpha^{\mathrm{n}} \mathrm{d}\left(\mathrm{gx_{0 }}, \mathrm{g} \mathrm{x}_{1}\right), \quad \forall \mathrm{n} \in \mathbb{N}_{0} .
$$

By classical technique, it can be easily shown that $\left\{g x_{n}\right\}$ is a Cauchy sequence. Thus, there is no need to use the condition (i) as we do not need to apply the contractivity condition on $d\left(g x_{m_{k}}, g x_{n_{k}}\right)$.

Further, as $\varphi(0)=0$, owing to Corollary 4.4 , we can remove condition (ii).

Remark 4.8. Notice that Theorem 4.7 improves Theorem 3.21 in the following respects:

- In the context of hypotheses (e), the completeness of $X$ is not necessary. Alternately, it can be replaced by the completeness of any subspace $Y$ satisfying $f(X) \subseteq Y$.

- In the context of hypotheses $\left(e^{\prime}\right)$, the completeness of the range subspaces $(f(X)$ or $g(X))$ are not necessary. Alternately, it can be replaced by the completeness of any subspace $Y$ satisfying $f(X) \subseteq$ $Y \subseteq g(X)$.

- The involved metrical terms namely: completeness, continuity, g-continuity and compatibility in Theorem 3.21 are not necessary as they can be alternately replaced by their respective " $\prec \succ$ analogues".

Using the similar arguments to Corollary 4.3, we have the following consequence of Theorem 4.7.

Corollary 4.9. Let $(\mathrm{X}, \mathrm{d}, \preceq)$ be $a \prec \succ$-complete ordered metric space and $\mathrm{f}$ and $\mathrm{g}$ two self-mappings on $\mathrm{X}$. Suppose that the following conditions hold:

(a) $f(X) \subseteq g(X)$;

(b) $\mathrm{f}$ is g-comparable;

(c) there exists $x_{0} \in X$ such that $\mathrm{g}\left(\mathrm{x}_{0}\right) \prec \succ \mathrm{f}\left(\mathrm{x}_{0}\right)$;

(d) there exists $\varphi \in \Omega$ such that

$$
\mathrm{d}(\mathrm{fx}, \mathrm{fy}) \leqslant \varphi(\mathrm{d}(\mathrm{gx}, \mathrm{gy})), \quad \forall x, y \in X
$$

with $\mathrm{g}(\mathrm{x}) \prec \succ \mathrm{g}(\mathrm{y})$; 
(e) (e1) f and g are $\prec \succ$-compatible;

(e2) g is $\prec \succ$-continuous;

(e3) either $\mathrm{f}$ is $\prec \succ$-continuous or $(\mathrm{X}, \mathrm{d}, \preceq)$ has $g$-TCC property,

or alternately

$\left(\mathrm{e}^{\prime}\right)\left(\mathrm{e}^{\prime} 1\right)$ there exists $a \prec \succ$-closed subspace $\mathrm{Y}$ of $\mathrm{X}$ such that $\mathrm{f}(\mathrm{X}) \subseteq \mathrm{Y} \subseteq \mathrm{g}(\mathrm{X})$;

$\left(e^{\prime} 2\right)$ either $f$ is $(g, \prec \succ)$-continuous or $f$ and $g$ are continuous or $(\mathrm{Y}, \mathrm{d}, \preceq)$ has TCC property.

Then $\mathrm{f}$ and $\mathrm{g}$ have a coincidence point.

Remark 4.10. If $\mathrm{g}$ is onto in Corollary 4.3 (also in Corollary 4.9), then we can drop assumption (a) as in this case it trivially holds. Also, we can remove assumption $\left(e^{\prime} 1\right)$ as it trivially holds for $Y=g(X)=X$ using Proposition 3.13. Whenever, $f$ is onto, owing to assumption (a), $g$ must be onto and hence again same conclusion is immediate.

On using Remarks 3.10, 3.12, 3.16, 3.18 and 3.20, we obtain the more natural versions of foregoing results in the form of the following consequence.

Corollary 4.11. Theorem 4.1 (also Theorem 4.7 and Corollaries 4.3, 4.4, 4.5 and 4.9) remains true, if the usual metrical terms namely: completeness, closedness, compatibility (or commutativity/weak commutativity), continuity and g-continuity are used instead of their respective " $\prec \succ$-analogues".

\section{Uniqueness results}

In this section, we formulate results ensuring the uniqueness of coincidence point, point of coincidence and common fixed point corresponding to Theorems 4.1 and 4.7. For a pair of self-mappings $f$ and $g$ defined on a nonempty set $X$ and a subset $E \subseteq X$, we denote the following sets:

$$
\begin{aligned}
C(f, g) & =\{x \in X: g x=f x\}, \text { i.e., the set of all coincidence points of } f \text { and } g, \\
\bar{C}(f, g) & =\{\bar{x} \in X: \bar{x}=g x=f x, x \in X\}, \text { i.e., the set of all points of coincidence of } f \text { and } g, \\
C(a, b, \prec \succ, E) & =\text { the class of all } \prec \succ-\text { chains between } a \text { and } b \text { in } E .
\end{aligned}
$$

Theorem 5.1. In addition to the hypotheses of Theorem 4.1 (also Theorem 4.7), suppose that the following condition holds:

$\left(\mathrm{u}_{1}\right) C(\mathrm{fx}, \mathrm{fy}, \prec \succ, \mathrm{gX})$ is nonempty, for each $\mathrm{x}, \mathrm{y} \in \mathrm{X}$.

Then $f$ and $g$ have a unique point of coincidence.

Proof. We prove the result for Theorem 4.1 and analogously, similar arguments can be used for Theorem 4.7. In view of Theorem $4.1, \bar{C}(f, g) \neq \emptyset$. Take $\bar{x}, \bar{y} \in \bar{C}(f, g)$, then there exist $x, y \in X$ such that

$$
\bar{x}=g(x)=f(x), \text { and } \bar{y}=g(y)=f(y) .
$$

Now, we show that $\bar{x}=\bar{y}$. As $f(x), f(y) \in f(X) \subseteq g(X)$, by $\left(u_{1}\right)$, there exists a $\prec \succ$-chain $\left\{g z_{1}, g z_{2}, \ldots, g z_{k}\right\}$ between $f(x)$ and $f(y)$ in $g(X)$, where $z_{1}, z_{2}, \ldots, z_{k} \in X$. Owing to (5.1), without loss of generality, we may choose $z_{1}=x$ and $z_{k}=y$. We have

$$
g\left(z_{\mathfrak{i}}\right) \prec \succ g\left(z_{\mathfrak{i}+1}\right), \quad \text { for each } i(1 \leqslant i \leqslant k-1) .
$$

Define the constant sequences $z_{\mathfrak{n}}^{1}=x$ and $z_{\mathfrak{n}}^{k}=y$, then using (5.1), we have $g\left(z_{n+1}^{1}\right)=f\left(z_{n}^{1}\right)=\bar{x}$ and $\mathrm{g}\left(z_{\mathfrak{n}+1}^{\mathrm{k}}\right)=\mathrm{f}\left(z_{\mathfrak{n}}^{\mathrm{k}}\right)=\overline{\mathrm{y}}$, for all $\mathrm{n} \in \mathbb{N}_{0}$. Put $z_{0}^{2}=z_{2}, z_{0}^{3}=z_{3}, \ldots, z_{0}^{\mathrm{k}-1}=z_{\mathrm{k}-1}$. Since $f(X) \subseteq \mathrm{g}(X)$, on 
the lines similar to that of Theorem 4.1 , we can define sequences $\left\{z_{\mathfrak{n}}^{2}\right\},\left\{z_{\mathfrak{n}}^{3}\right\}, \ldots,\left\{z_{\mathfrak{n}}^{k-1}\right\}$ in $X$ such that $g\left(z_{\mathfrak{n}+1}^{2}\right)=f\left(z_{\mathfrak{n}}^{2}\right), g\left(z_{n+1}^{3}\right)=f\left(z_{\mathfrak{n}}^{3}\right), \ldots, g\left(z_{n+1}^{k-1}\right)=f\left(z_{n}^{k-1}\right)$ for all $n \in \mathbb{N}_{0}$. Hence, we have

$$
g\left(z_{\mathfrak{n}+1}^{i}\right)=f\left(z_{\mathfrak{n}}^{i}\right), \quad \forall n \in \mathbb{N}_{0} \text { and for each } i(1 \leqslant i \leqslant k) .
$$

Now we claim that

$$
\mathrm{g}\left(z_{\mathfrak{n}}^{\mathrm{i}}\right) \prec \succ \mathrm{g}\left(z_{\mathrm{n}}^{\mathrm{i}+1}\right), \quad \forall \mathrm{n} \in \mathbb{N}_{0} \text { and for each } \mathrm{i}(1 \leqslant i \leqslant k-1) .
$$

We prove this fact by the method of mathematical induction. In view of (5.2), (5.4) holds for $n=0$. Suppose that (5.4) holds for $n=r>0$, i.e.,

$$
\mathrm{g}\left(z_{\mathrm{r}}^{\mathrm{i}}\right) \prec \succ \mathrm{g}\left(z_{\mathrm{r}}^{\mathrm{i}+1}\right), \quad \text { for each } i(1 \leqslant i \leqslant k-1) .
$$

On using g-comparability of $f$, we obtain

$$
f\left(z_{r}^{i}\right) \prec \succ f\left(z_{r}^{i+1}\right), \quad \text { for each } i(1 \leqslant i \leqslant k-1),
$$

which on using (5.3), gives rise

$$
g\left(z_{r+1}^{i}\right) \prec \succ g\left(z_{r+1}^{i+1}\right), \quad \text { for each } i(1 \leqslant i \leqslant k-1) .
$$

It follows that (5.4) holds for $n=r+1$. Thus, by induction, (5.4) holds for all $n \in \mathbb{N}_{0}$. Now for each $n \in \mathbb{N}_{0}$ and for each $i(1 \leqslant i \leqslant k-1)$, define $t_{\mathfrak{n}}^{i}:=d\left(g z_{n}^{i}, g z_{n}^{i+1}\right)$. We claim that

$$
\lim _{n \rightarrow \infty} t_{n}^{i}=0, \quad \text { for each } i(1 \leqslant i \leqslant k-1) .
$$

On fixing $i$, the two cases arise. Firstly, suppose that $t_{\mathfrak{n}_{0}}^{i}=d\left(g z_{\mathfrak{n}_{0}}^{i} g z_{\mathfrak{n}_{0}}^{i+1}\right)=0$ for some $n_{0} \in \mathbb{N}_{0}$, then by g-admissibility of $f$, we obtain $d\left(f z_{\mathfrak{n}_{0}}^{\mathfrak{i}}, f z_{\mathfrak{n}_{0}}^{i+1}\right)=0$. Consequently on using (5.4), we get

$$
\mathrm{t}_{\mathfrak{n}_{0}+1}^{\mathrm{i}}=\mathrm{d}\left(\mathrm{g} z_{\mathfrak{n}_{0}+1}^{\mathrm{i}}, \mathrm{g} z_{\mathfrak{n}_{0}+1}^{\mathrm{i}+1}\right)=\mathrm{d}\left(\mathrm{f} z_{\mathfrak{n}_{0},}^{\mathrm{i}}, \mathrm{f} z_{\mathfrak{n}_{0}}^{\mathrm{i}+1}\right)=0 .
$$

Thus by induction, we get $t_{n}^{i}=0$, for all $n \geqslant n_{0}$, yielding thereby $\lim _{n \rightarrow \infty} t_{n}^{i}=0$. On the other hand, suppose that $t_{n}>0$, for all $n \in \mathbb{N}_{0}$. Then, on using (5.3), (5.4) and assumption (e), we have

$$
\begin{aligned}
\mathrm{t}_{\mathrm{n}+1}^{\mathrm{i}} & =\mathrm{d}\left(\mathrm{g} z_{\mathrm{n}+1}^{\mathrm{i}}, \mathrm{g} z_{\mathrm{n}+1}^{\mathrm{i}+1}\right) \\
& =\mathrm{d}\left(\mathrm{f} z_{\mathfrak{n}}^{\mathrm{i}}, \mathrm{f} z_{\mathrm{n}}^{\mathrm{i}+1}\right) \\
& \leqslant \varphi\left(\mathrm{d}\left(\mathrm{g} z_{\mathfrak{n}}^{\mathrm{i}}, z_{\mathrm{n}}^{\mathrm{i}+1}\right)\right) \\
& =\varphi\left(\mathrm{t}_{\mathfrak{n}}^{\mathrm{i}}\right),
\end{aligned}
$$

so that

$$
t_{n+1}^{i} \leqslant \varphi\left(t_{n}^{i}\right) .
$$

Hence on applying Lemma 2.7, we obtain $\lim _{n \rightarrow \infty} t_{n}^{i}=0$. Thus, in both the cases, (5.5) holds for each $i(1 \leqslant i \leqslant k-1)$. On using triangular inequality and (5.5), we obtain

$$
d(\bar{x}, \bar{y}) \leqslant t_{n}^{1}+t_{n}^{2}+\cdots+t_{n}^{k-1} \rightarrow 0, \text { as } n \rightarrow \infty,
$$

so that

$$
\bar{x}=\bar{y} .
$$

Corollary 5.2. Theorem 5.1 remains true, if we replace the condition $\left(\mathrm{u}_{1}\right)$ by one of the following conditions (besides retaining rest of the hypotheses): 
$\left(\mathrm{u}_{1}^{1}\right)(\mathrm{fX}, \preceq)$ is totally ordered;

$\left(\mathrm{u}_{1}^{2}\right)(\mathrm{X}, \preceq)$ is $(\mathrm{f}, \mathrm{g})$-directed.

Proof. Suppose that $\left(\mathrm{u}_{1}^{1}\right)$ holds, then for each pair $x, y \in X$, we have

$$
f(x) \prec \succ f(y),
$$

which implies that $\{f x, f y\}$ is a $\prec \succ$-chain between $f(x)$ and $f(y)$ in $g(X)$ so that $C(f x, f y, \prec \succ, g X)$ is nonempty, for each $x, y \in X$, i.e., $\left(u_{1}\right)$ holds and hence Theorem 5.1 is applicable. Next, assume that $\left(u_{1}^{2}\right)$ holds, then for each pair $x, y \in X$, there exists a $z \in X$ such that

$$
f(x) \prec \succ g(z) \prec \succ f(y),
$$

which implies that $\{f x, g z, f y\}$ is a $\prec \succ$-chain between $f(x)$ and $f(y)$ in $g(X)$ so that $C(f x, f y, \prec \succ, g X)$ is nonempty, for each $x, y \in X$, i.e., $\left(u_{1}\right)$ holds and hence Theorem 5.1 is applicable.

Theorem 5.3. In addition to the hypotheses of Theorem 5.1, suppose that the following condition holds:

$\left(\mathrm{u}_{2}\right)$ one of $\mathrm{f}$ and $\mathrm{g}$ is one-one.

Then $f$ and $g$ have a unique coincidence point.

Proof. Take $x, y \in C(f, g)$, then in view of Theorem 5.1, we have

$$
g(x)=f(x)=f(y)=g(y) .
$$

As $f$ or $g$ is one-one, we have

$$
x=y .
$$

Theorem 5.4. In addition to the hypotheses embodied in condition $\left(\mathrm{e}^{\prime}\right)$ of Theorem 5.1, suppose that the following condition holds:

$\left(e^{\prime} 3\right)$ f and $g$ are weakly compatible.

Then $\mathrm{f}$ and $\mathrm{g}$ have a unique common fixed point.

Proof. Owing to Remark 3.20 as well as assumption ( $\left.\mathrm{e}^{\prime} 3\right)$, the mappings $f$ and $g$ are weakly compatible. Let $x$ be a coincidence point of $f$ and $g$. Write $g(x)=f(x)=\bar{x}$, then in view of Lemma 2.10, $\bar{x}$ is also a coincidence point of $f$ and $g$. It follows from Theorem 5.1 with $y=\bar{x}$ that $g(x)=g(\bar{x})$, i.e., $\bar{x}=g(\bar{x})$, which yields that

$$
\bar{x}=g(\bar{x})=f(\bar{x}) .
$$

Hence, $\bar{x}$ is a common fixed point of $f$ and $g$. To prove uniqueness, assume that $x^{*}$ is another common fixed point of $f$ and $g$. Then again from Theorem 5.1, we have

$$
x^{*}=g\left(x^{*}\right)=g(\bar{x})=\bar{x} .
$$

This completes proof.

\section{Fixed point theorems}

On setting $\mathrm{g}=\mathrm{I}$, the identity mapping on $\mathrm{X}$, in foregoing results, we get the following corresponding fixed point theorems.

Theorem 6.1. Let $(\mathrm{X}, \mathrm{d}, \preceq)$ be an ordered metric space and $\mathrm{f}$ a self-mapping on $\mathrm{X}$. Let $\mathrm{Y}$ be $a \prec \succ$-complete subspace 
of $\mathrm{X}$ such that $\mathrm{f}(\mathrm{X}) \subseteq \mathrm{Y}$ and $(\mathrm{Y}, \preceq)$ is sequentially chainable. Suppose that the following conditions hold:

(i) $\mathrm{f}$ is comparable;

(ii) either $\mathrm{f}$ is $\prec \succ$-continuous or $(\mathrm{Y}, \mathrm{d}, \preceq)$ has TCC property;

(iii) there exists $x_{0} \in X$ such that $x_{0} \prec \succ f\left(x_{0}\right)$;

(iv) there exists $\varphi \in \Omega$ such that

$$
d(f x, f y) \leqslant \varphi(d(x, y)), \quad \forall x, y \in X
$$

with $x \prec \succ y$.

Then $\mathrm{f}$ has a fixed point.

Corollary 6.2. Let $(\mathrm{X}, \mathrm{d}, \preceq)$ be $a \prec \succ$-complete ordered metric space such that $(\mathrm{X}, \preceq)$ is sequentially chainable and $\mathrm{f}$ a self-mapping on $\mathrm{X}$. Suppose that the following conditions hold:

(i) $\mathrm{f}$ is comparable;

(ii) either $\mathrm{f}$ is $\prec \succ$-continuous or $(\mathrm{X}, \mathrm{d}, \preceq)$ has TCC property;

(iii) there exists $\mathrm{x}_{0} \in \mathrm{X}$ such that $\mathrm{x}_{0} \prec \succ \mathrm{f}\left(\mathrm{x}_{0}\right)$;

(iv) there exists $\varphi \in \Omega$ such that

$$
\mathrm{d}(\mathrm{f} x, \mathrm{fy}) \leqslant \varphi(\mathrm{d}(\mathrm{x}, \mathrm{y})), \quad \forall x, y \in X
$$

with $x \prec \succ y$.

Then $\mathrm{f}$ has a fixed point.

Theorem 6.3. Let $(\mathrm{X}, \mathrm{d}, \preceq)$ be an ordered metric space and $\mathrm{f}$ a self-mapping on $\mathrm{X}$. Let $\mathrm{Y}$ be $a \prec \succ$-complete subspace of $X$ such that $f(X) \subseteq Y$. Suppose that the following conditions hold:

(i) $\mathrm{f}$ is comparable;

(ii) either $\mathrm{f}$ is $\prec \succ$-continuous or $(\mathrm{Y}, \mathrm{d}, \preceq)$ has TCC property;

(iii) there exists $x_{0} \in X$ such that $x_{0} \prec \succ f\left(x_{0}\right)$;

(iv) there exists $\alpha \in[0,1)$ such that

$$
d(f x, f y) \leqslant \alpha d(x, y), \quad \forall x, y \in X
$$

with $x \prec \succ y$.

Then $\mathrm{f}$ has a fixed point.

Corollary 6.4. Let $(\mathrm{X}, \mathrm{d}, \preceq)$ be $a \prec \succ$-complete ordered metric space and $\mathrm{f}$ a self-mapping on $\mathrm{X}$. Suppose that the following conditions hold:

(i) $\mathrm{f}$ is comparable;

(ii) either $\mathrm{f}$ is $\prec \succ$-continuous or $(\mathrm{X}, \mathrm{d}, \preceq)$ has TCC property;

(iii) there exists $x_{0} \in X$ such that $x_{0} \prec \succ f\left(x_{0}\right)$;

(iv) there exists $\alpha \in[0,1)$ such that

$$
d(f x, f y) \leqslant \alpha d(x, y), \quad \forall x, y \in X,
$$

with $x \prec \succ y$. 
Then $\mathrm{f}$ has a fixed point.

Theorem 6.5. In addition to the hypotheses of Theorem 6.1 (also Theorem 6.3), suppose that the following condition holds:

(u) $C(f x, f y, \prec \succ)$ is nonempty for each $x, y \in X$.

Then $\mathrm{f}$ has a unique fixed point.

Corollary 6.6. Theorem 6.5 remains true, if we replace the condition $(\mathrm{u})$ by one of the following conditions:

$\left(\mathbf{u}^{1}\right)(\mathrm{fX}, \preceq)$ is totally ordered;

$\left(\mathbf{u}^{2}\right)(\mathbf{X}, \preceq)$ is f-directed.

\section{Examples}

In this section, we furnish some examples illustrating our newly proved results.

Example 7.1. Consider the set of real numbers $\mathbb{R}$ equipped with usual metric d. Define a partial order $\preceq$ on $\mathbb{R}$ by $x \preceq y \Leftrightarrow|x| \leqslant|y|$ and $x y \geqslant 0$. Then $(\mathbb{R}, d, \preceq)$ is a $\prec \succ$-complete ordered metric space. Define $f, g: \mathbb{R} \rightarrow \mathbb{R}$ by $f(x)=\frac{x^{2}}{6}$ and $g(x)=-x^{2}$ for all $x \in \mathbb{R}$. Then $f$ is $g$-comparable. Define $\varphi:[0, \infty) \rightarrow[0, \infty)$ by $\varphi(t)=\frac{t}{4}$, for all $t \in[0, \infty)$, then $\varphi \in \Omega$. Now, for $x, y \in \mathbb{R}$ with $g(x) \preceq g(y)$, we have

$$
d(f x, f y)=\left|\frac{x^{2}}{6}-\frac{y^{2}}{6}\right|=\frac{1}{6}\left|x^{2}-y^{2}\right|=\frac{1}{6} d(g x, g y)<\frac{1}{4} d(g x, g y)=\varphi(d(g x, g y)) .
$$

Thus, $f, g$ and $\varphi$ satisfy assumption (d) of Theorem 4.1. By a routine calculation, one can also verify other conditions mentioned in (e) (of Theorem 4.1). Thus, all the conditions of Theorem 4.1 are satisfied (with $Y=X)$, so that $f$ and $g$ have a coincidence point in $\mathbb{R}$. As $\left(u_{1}\right)$ also holds, therefore owing to Theorem 5.1, $f$ and $g$ have a unique point of coincidence (namely: $\bar{x}=0$ ). Furthermore, In view of Theorem $5.4, f$ and $g$ have a unique common fixed point (namely: $x=0$ ).

Example 7.2. Consider $X=\mathbb{R}$ equipped with usual metric $d$ and usual (partial) order $\preceq$. Define $f, g: X \rightarrow$ $X$ by $f(x)=9$ and $g(x)=x^{2}-7$, for all $x \in X$. Then $f$ is $g$-comparable. Let $\varphi \in \Omega$ be arbitrary. Then for $x, y \in X$ with $g(x) \preceq g(y)$, we have

$$
\mathrm{d}(\mathrm{fx}, \mathrm{fy})=|9-9|=0 \leqslant \varphi\left(\left|x^{2}-\mathrm{y}^{2}\right|\right)=\varphi(\mathrm{d}(\mathrm{gx}, \mathrm{gy})),
$$

so that $f, g$ and $\varphi$ satisfy the assumption (d) (of Theorem 4.1). Also, the mappings $f$ and $g$ are not $\prec \succ$ compatible and hence (e) does not hold. But the subspace $Y:=g(X)=[-7, \infty)$ is $\prec \succ$-complete while $f$ and $g$ are continuous so that all the conditions mentioned in $\left(\mathrm{e}^{\prime}\right)$ are satisfied. Therefore, by Theorem 4.1, $f$ and $g$ have a coincidence point in $X$. As $\left(u_{1}\right)$ also holds, owing to Theorem 5.1, $f$ and $g$ have a unique point of coincidence (namely: $\bar{x}=9$ ). Notice that neither $f$ nor $g$ is one-one (i.e., $\left(u_{2}\right)$ does not hold) so that Theorem 5.3 cannot be applied, which guarantees the uniqueness of coincidence point. Observe that there are two coincidence points (namely: $x=4$ and $x=-4$ ). Also, $f$ and $g$ are not weakly compatible (i.e., ( $\left.e^{\prime} 3\right)$ does not hold) and hence, we can not apply Theorem 5.4, which ensures the uniqueness of common fixed point. Notice that there is no common fixed point of $f$ and $g$.

Example 7.3. Consider $X=[0,2]$. Then $(X, d, \preceq)$ is a $\prec \succ$-complete ordered metric space under the usual metric $d$ and the usual (partial) order $\preceq$. Define $f: X \rightarrow X$ by $f(x)=x-\frac{1}{2} \chi^{2}$, then $f$ is comparable but not monotone. Define $\varphi:[0, \infty) \rightarrow[0, \infty)$ by $\varphi(t)=t-\frac{1}{2} t^{2}$, for all $t \in[0, \infty)$, then $\varphi \in \Omega$. Also, for $x, y \in X$ with $x \prec \succ y$, we have

$$
d(f x, f y)=|x-y|\left|1-\frac{1}{2}(x+y)\right|
$$




$$
\begin{aligned}
& \leqslant|x-y|\left|1-\frac{1}{2}\right| x-y|| \\
& \leqslant|x-y|-\frac{1}{2}|x-y|^{2} \\
& =\varphi(d(x, y)),
\end{aligned}
$$

so that

$$
d(f x, f y) \leqslant \varphi(d(x, y)) .
$$

i.e., $f$ satisfies the contractivity condition (iv) of Corollary 6.2. Thus, all the conditions mentioned in Corollary 6.2 and Theorem 6.5 are satisfied and hence $f$ has a unique fixed point in $X$ (namely: $x=0$ ).

Suppose there exists $\alpha \in[0,1)$ such that

$$
d(f x, f y) \leqslant \alpha d(x, y)
$$

Then, for $x \neq y$ with $x \prec \succ y$, we have

$$
\begin{aligned}
\left|x-\frac{1}{2} x^{2}-y+\frac{1}{2} y^{2}\right| & \leqslant \alpha|x-y| \\
\Rightarrow\left|(x-y)-\frac{1}{2}\left(x^{2}-y^{2}\right)\right| & \leqslant \alpha|x-y| \\
\Rightarrow|x-y|\left|1-\frac{1}{2}(x+y)\right| & \leqslant \alpha|x-y| \\
\Rightarrow\left|1-\frac{1}{2}(x+y)\right| & \leqslant \alpha,
\end{aligned}
$$

so that for $x, y$ small enough $\alpha \rightarrow 1$, which yields that there is no $\alpha \in[0,1)$ such that contractivity condition (7.1) holds, i.e., $f$ is not comparable linear contraction. Henceforth Corollary 6.4 (i.e., comparable Banach contraction principle) is not applicable in the context of present example.

\section{An application to integral equation}

In this section, using certain results (particularly, Corollary 6.2 and Theorem 6.5), we study the existence and uniqueness of solution of the following integral equation:

$$
\mathrm{u}(\mathrm{t})=\int_{0}^{T} \mathrm{M}(\mathrm{t}, \xi, \mathrm{u}(\xi)) \mathrm{d} \xi, \quad \forall \mathrm{t} \in \mathrm{I},
$$

where $T>0, I=[0, T], u: I \rightarrow \mathbb{R}$ is unknown function and $M: I \times I \times \mathbb{R} \rightarrow \mathbb{R}$ is known function.

We denote $\mathcal{C}(S)$ by the space of all real-valued continuous functions on a nonempty set $S$.

Definition 8.1. A function $\eta \in \mathcal{C}(I)$ is called a lower solution of (8.1), if

$$
\eta(t) \leqslant \int_{0}^{T} M(t, \xi, \eta(\xi)) d \xi, \quad \forall t \in I .
$$

Definition 8.2. A function $\eta \in \mathcal{C}(\mathrm{I})$ is called an upper solution of (8.1), if

$$
\eta(t) \geqslant \int_{0}^{T} M(t, \xi, \eta(\xi)) d \xi, \quad \forall t \in I .
$$

Let $\mathfrak{F}$ denote the family of functions $\phi:[0, \infty] \rightarrow[0, \infty]$ satisfying the following conditions:

(i) $\phi$ is continuous and increasing; 
(ii) $\phi(t)<t$ for each $t>0$.

Typical examples of $\mathfrak{F}$ are $\phi(t)=\alpha . t, 0 \leqslant \alpha<1, \phi(t)=\frac{t}{1+t}$ and $\phi(t)=\ln (1+t)$. Also, clearly $\mathfrak{F} \subset \Omega$.

Now, we prove the following result on the existence and uniqueness of the solution of the problem described by (8.1) in the presence of a lower solution (or an upper solution).

Theorem 8.3. In respect of the problem described by (8.1), suppose that the following assumptions hold:

(a) $M \in \mathcal{C}(I \times I \times \mathbb{R})$ and $M(t, \xi, x) \geqslant 0, \forall t, \xi \in I, x \in \mathbb{R}$;

(b) there exists $\phi \in \mathfrak{F}$ such that for all $\mathrm{t}, \xi \in \mathrm{I}$ and for all $\mathrm{x}, \mathrm{y} \in \mathbb{R}$ with $\mathrm{x} \leqslant \mathrm{y}$,

$$
0 \leqslant M(t, \xi, x)-M(t, \xi, y) \leqslant p(t, \xi) \phi(y-x),
$$

where $\mathrm{p}: \mathrm{I} \times \mathrm{I} \rightarrow[0, \infty)$ is a continuous function satisfying

$$
\sup _{t \in \mathrm{I}} \int_{0}^{T} p(t, \xi) d \xi \leqslant 1 .
$$

Then the existence of a lower solution (or an upper solution) of the problem (8.1) ensures the existence and uniqueness of the solution of this problem.

Proof. Define a function $\mathcal{A}: \mathcal{C}(\mathrm{I}) \rightarrow \mathcal{C}(\mathrm{I})$ by

$$
(\mathcal{A u})(\mathrm{t})=\int_{0}^{\mathrm{T}} \mathrm{M}(\mathrm{t}, \xi, \mathrm{u}(\xi)) \mathrm{d} \xi, \quad \forall \mathrm{t} \in \mathrm{I} .
$$

Clearly, if $u \in \mathcal{C}(\mathrm{I})$ is a fixed point of $\mathcal{A}$ then $u$ is a solution of (8.1). On $\mathcal{C}(\mathrm{I})$, define a metric $\mathrm{d}$ given by:

$$
\mathrm{d}(\mathrm{u}, v)=\sup _{\mathrm{t} \in \mathrm{I}}|\mathrm{u}(\mathrm{t})-v(\mathrm{t})|, \quad \forall \mathfrak{u}, v \in \mathcal{C}(\mathrm{I}) .
$$

On $\mathcal{C}(\mathrm{I})$, define a partial order $\preceq$ given by:

$$
\mathrm{u}, v \in \mathcal{C}(\mathrm{I}) ; \mathrm{u} \preceq v \Longleftrightarrow \mathrm{u}(\mathrm{t}) \leqslant v(\mathrm{t}), \quad \forall \mathrm{t} \in \mathrm{I} .
$$

Now, we check that all the conditions of Corollary 6.2 are satisfied. Clearly, ( $(I), d, \preceq)$ is a $\prec \succ$-complete ordered metric space and $(\mathcal{C}(\mathrm{I}), \preceq)$ is sequentially chainable.

(i) Take $u, v \in \mathcal{C}(\mathrm{I})$ such that $u \prec \succ v$, then by (8.4), we obtain

$$
u(\xi) \leqslant v(\xi), \quad \forall \xi \in I \quad \text { or } u(\xi) \geqslant v(\xi), \quad \forall \xi \in I,
$$

which, for each $\mathrm{t} \in \mathrm{I}$, using assumption (b) gives rise

$$
M(t, \xi, u(\xi)) \geqslant M(t, \xi, v(\xi)), \quad \forall \xi \in I \quad \text { or } M(t, \xi, u(\xi)) \leqslant M(t, \xi, v(\xi)), \quad \forall \xi \in I .
$$

On using (8.2), (8.5) and assumption (a), we get

$$
\begin{aligned}
(\mathcal{A u})(\mathrm{t}) & =\int_{0}^{T} \mathrm{M}(\mathrm{t}, \xi, \mathrm{u}(\xi)) \mathrm{d} \xi \\
& \geqslant \int_{0}^{T} \mathrm{M}(\mathrm{t}, \xi, v(\xi)) \mathrm{d} \xi \\
& =(\mathcal{A} v)(\mathrm{t}), \quad \forall \mathrm{t} \in \mathrm{I},
\end{aligned}
$$

or

$$
\begin{aligned}
(\mathcal{A u})(\mathrm{t}) & =\int_{0}^{T} \mathrm{M}(\mathrm{t}, \xi, \mathrm{u}(\xi)) \mathrm{d} \xi \\
& \leqslant \int_{0}^{T} \mathrm{M}(\mathrm{t}, \xi, v(\xi)) \mathrm{d} \xi \\
& =(\mathcal{A} v)(\mathrm{t}) \quad \forall \mathrm{t} \in \mathrm{I},
\end{aligned}
$$

which owing to (8.4) implies that $\mathcal{A}(u) \prec \succ \mathcal{A}(v)$ so that $\mathcal{A}$ is comparable. 
(ii) Take a sequence $\left\{u_{n}\right\} \subset \mathcal{C}(I)$ such that $u_{n} \uparrow u \in \mathcal{C}(I)$. Then for each $t \in I,\left\{u_{n}(t)\right\}$ is a sequence in $\mathbb{R}$ converging to $u(t)$. Hence, $\left\{u_{n}(t)\right\}$ has a monotone subsequence $\left\{u_{n_{k}}(t)\right\}$. Therefore, for all $k \in \mathbb{N}_{0}$ and for all $t \in I$, we have

$$
\begin{array}{ll}
u_{n_{k}}(t) \leqslant u(t), & \text { if }\left\{u_{n_{k}}(t)\right\} \text { is increasing, } \\
u_{n_{k}}(t) \geqslant u(t), & \text { if }\left\{u_{n_{k}}(t)\right\} \text { is decreasing, }
\end{array}
$$

which by using (8.4) implies that $u_{n_{k}} \prec \succ u$, for all $k \in \mathbb{N}_{0}$ so that $(\mathcal{C}(I), d, \preceq)$ has TCC property.

(iii) If $\eta \in \mathcal{C}(\mathrm{I})$ is a lower (resp. an upper) solution of (8.1), then using (8.2) and (8.4), we can verify that $\eta \preceq \mathcal{A}(\eta)$ (resp. $\eta \succeq \mathcal{A}(\eta)$ ). Hence, in both the cases, we have $\eta \prec \succ \mathcal{A}(\eta)$, for some lower or upper solution $\eta$.

(iv) Take $u, v \in \mathcal{C}(\mathrm{I})$ such that $u \preceq v$. On using (8.2), (8.3) and assumption (b), we obtain

$$
\begin{aligned}
d(\mathcal{A u}, \mathcal{A} v) & =\sup _{\mathrm{t} \in \mathrm{I}}|(\mathcal{A u})(\mathrm{t})-(\mathcal{A} v)(\mathrm{t})|=\sup _{\mathrm{t} \in \mathrm{I}}\left|\int_{0}^{T} M(t, \xi, u(\xi)) d \xi-\int_{0}^{T} M(t, \xi, v(\xi)) d \xi\right| \\
& =\sup _{\mathrm{t} \in \mathrm{I}} \int_{0}^{T}(M(t, \xi, u(\xi))-M(t, \xi, v(\xi))) d \xi \\
& \leqslant \sup _{t \in \mathrm{I}} \int_{0}^{T} p(t, \xi) \phi(v(\xi)-u(\xi)) d \xi .
\end{aligned}
$$

Given that $\phi$ is increasing on $[0, \infty)$ and $u \preceq v$, which implies that $\phi(v(\xi)-u(\xi)) \leqslant \phi(d(u, v))$ for all $\xi \in \mathrm{I}$. Hence, (8.6) reduces to

$$
d(\mathcal{A u}, \mathcal{A v}) \leqslant \phi(d(u, v)) \sup _{t \in \mathrm{I}} \int_{0}^{T} p(t, \xi) d \xi,
$$

which again using assumption (b) gives rise

$$
\mathrm{d}(\mathcal{A u}, \mathcal{A} v) \leqslant \phi(\mathrm{d}(\mathrm{u}, v)), \quad \forall u, v \in \mathcal{C}(\mathrm{I}) \text { such that } u \preceq v,
$$

where $\phi \in \mathfrak{F} \subset \Omega$.

Thus, all the conditions of Corollary 6.2 are satisfied, which ensures that $\mathcal{A}$ has a fixed point.

Finally, choose arbitrary $u, v \in \mathcal{C}(\mathrm{I})$ and write $w:=\max \{\mathcal{A} u, \mathcal{A} v\} \in \mathcal{C}(\mathrm{I})$. As $\mathcal{A}(\mathfrak{u}) \preceq \mathfrak{w}$ and $\mathcal{A}(v) \preceq w$, $\{\mathcal{A u}, w, \mathcal{A} v\}$ is a $\prec \succ$-chain between $\mathcal{A}(u)$ and $\mathcal{A}(v)$. Now, in view of Theorem $6.5, \mathcal{A}$ has a unique fixed point, which is, indeed, a unique solution of the problem described by (8.1).

\section{Conclusion}

In an attempt to extend Theorem 3.21 from linear contractions to Boyd-Wong type nonlinear contractions, we were compelled to add two extra conditions in Theorem 4.1 (namely: sequential chainability and g-admissibility), which substantiate the utility of this extension. As per new work, readers may attempt to prove such results under various well-known contractions such as: quasi contractions, Matkowski type contractions, weak nonlinear contractions, rational type contractions, Meir-Keeler type contractions, cyclic contractions, Geraghty-type contractions etc. besides using implicit relations.

\section{Acknowledgment}

All the authors are thankful to an anonymous learned referee for his valuable suggestions and fruitful comments. The second author is also grateful to University Grant Commission, Government of India, New Delhi, India for providing financial assistance under start up grant. 


\section{References}

[1] R. P. Agarwal, M. A. El-Gebeily, D. O’Regan, Generalized contractions in partially ordered metric spaces, Appl. Anal., 87 (2008), 109-116. 1

[2] A. Alam, M. Imdad, Comparable linear contractions in ordered metric spaces, ArXiv, 2015 (2015), 23 pages. 1, 3.1, 3, 3.7, 3, 3.8, 3, 3.21

[3] A. Alam, M. Imdad, Monotone generalized contractions in ordered metric spaces, Bull. Korean Math. Soc., 53 (2016), 61-81. 1, 3, 3.2, 3.3, 3.4, 3, 3.5, 3.6, 4.6

[4] A. Alam, A. R. Khan, M. Imdad, Some coincidence theorems for generalized nonlinear contractions in ordered metric spaces with applications, Fixed Point Theory Appl., 2014 (2014), 30 pages. 1, 1.1, 1, 2.7, 2.10

[5] A. Alam, Q. H. Khan, M. Imdad, Discussion on some recent order-theoretic metrical coincidence theorems involving nonlinear contractions, J. Funct. Spaces, 2016 (2016), 11 pages. 2.1

[6] I. Altun, H. Simsek, Some fixed point theorems on ordered metric spaces and application, Fixed Point Theory Appl., 2010 (2010), 17 pages. 1

[7] A. Amini-Harandi, H. Emami, A fixed point theorem for contraction type maps in partially ordered metric spaces and application to ordinary differential equations, Nonlinear Anal., 72 (2010), 2238-2242. 1

[8] M. Berzig, E. Karapınar, A. F. Roldán-López-de-Hierro, Discussion on generalized- $(\alpha \psi, \beta \phi)$-contractive mappings via generalized altering distance function and related fixed point theorems, Abstr. Appl. Anal., 2014 (2014), 12 pages. 2.8

[9] D. W. Boyd, J. S. W. Wong, On nonlinear contractions, Proc. Amer. Math. Soc., 20 (1969), 458-464. 1, 1

[10] F. E. Browder, On the convergence of successive approximations for nonlinear functional equations, Akad. Wetensch. Proc. Ser. A 71=Indag. Math., 30 (1968), 27-35. 1

[11] J. Caballero, J. Harjani, K. Sadarangani, Contractive-like mapping principles in ordered metric spaces and application to ordinary differential equations, Fixed Point Theory Appl., 2010 (2010), 14 pages. 1

[12] L. Ćirić, N. Cakić, M. Rajović, J. S. Ume, Monotone generalized nonlinear contractions in partially ordered metric spaces, Fixed Point Theory Appl., 2008 (2008), 11 pages. 1

[13] D. Dorić, Z. Kadelburg, S. Radenović, P. Kumam, A note on fixed point results without monotone property in partially ordered metric space, Rev. R. Acad. Cienc. Exactas Fs. Nat. Ser. A Math. RACSAM, 108 (2014), 503-510. 1

[14] R. H. Haghi, S. Rezapour, N. Shahzad, Some fixed point generalizations are not real generalizations, Nonlinear Anal., 74 (2011), 1799-1803. 2.9

[15] J. Harjani, K. Sadarangani, Fixed point theorems for weakly contractive mappings in partially ordered sets, Nonlinear Anal., 71 (2009), 3403-3410. 1

[16] J. Harjani, K. Sadarangani, Generalized contractions in partially ordered metric spaces and applications to ordinary differential equations, Nonlinear Anal., 72 (2010), 1188-1197.

[17] J. Jachymski, Equivalent conditions for generalized contractions on (ordered) metric spaces, Nonlinear Anal., 74 (2011), 768-774.

[18] M. Jleli, V. Čojbašić Rajić, B. Samet, C. Vetro, Fixed point theorems on ordered metric spaces and applications to nonlinear elastic beam equations, J. Fixed Point Theory Appl., 12 (2012), 175-192. 1, 2.8, 3

[19] N. Jotić, Some fixed point theorems in metric spaces, Indian J. Pure Appl. Math., 26 (1995), 947-952. 1

[20] G. Jungck, Commuting mappings and fixed points, Amer. Math. Monthly, 83 (1976), 261-263. 2.2

[21] G. Jungck, Compatible mappings and common fixed points, Internat. J. Math. Math. Sci., 9 (1986), 771-779. 2.3

[22] G. Jungck, Common fixed points for noncontinuous nonself maps on nonmetric spaces, Far East J. Math. Sci., 4 (1996), 199-215. 2.2

[23] V. Lakshmikantham, L. Ćirić, Coupled fixed point theorems for nonlinear contractions in partially ordered metric spaces, Nonlinear Anal., 70 (2009), 4341-4349. 1

[24] A. Mukherjea, Contractions and completely continuous mappings, Nonlinear Anal., 1 (1976/77), 235-247. 1

[25] H. K. Nashine, I. Altun, A common fixed point theorem on ordered metric spaces, Bull. Iranian Math. Soc., 38 (2012), 925-934. 1

[26] J. J. Nieto, R. Rodríguez-López, Contractive mapping theorems in partially ordered sets and applications to ordinary differential equations, Order, 22 (2005), 223-239. 1

[27] J. J. Nieto, R. Rodríguez-López, Existence and uniqueness of fixed point in partially ordered sets and applications to ordinary differential equations, Acta Math. Sin. (Engl. Ser.), 23 (2007), 2205-2212. 1, 3

[28] D. O'Regan, A. Petruşel, Fixed point theorems for generalized contractions in ordered metric spaces, J. Math. Anal. Appl., 341 (2008), 1241-1252. 1

[29] A. C. M. Ran, M. C. B. Reurings, A fixed point theorem in partially ordered sets and some applications to matrix equations, Proc. Amer. Math. Soc., 132 (2004), 1435-1443. 1

[30] K. P. R. Sastry, I. S. R. Krishna Murthy, Common fixed points of two partially commuting tangential selfmaps on a metric space, J. Math. Anal. Appl., 250 (2000), 731-734. 2.4

[31] S. Sessa, On a weak commutativity condition of mappings in fixed point considerations, Publ. Inst. Math. (Beograd) (N.S.), 32 (1982), 149-153. 2.3

[32] M. Turinici, Abstract comparison principles and multivariable Gronwall-Bellman inequalities, J. Math. Anal. Appl., 117 (1986), 100-127. 1

[33] M. Turinici, Fixed points for monotone iteratively local contractions, Demonstratio Math., 19 (1986), 171-180. 1 
[34] M. Turinici, Ran-Reurings fixed point results in ordered metric spaces, Libertas Math., 31 (2011), 49-55. 1, 3

[35] M. Turinici, Nieto-Lopez theorems in ordered metric spaces, Math. Student, 81 (2012), 219-229. 1, 3

[36] M. Turinici, Contractive operators in relational metric spaces, Handbook of functional equations, Springer Optim. Appl., Springer, New York, 95 (2014), 419-458. 2.8

[37] J. Wu, Y.-C. Liu, Fixed point theorems for monotone operators and applications to nonlinear elliptic problems, Fixed Point Theory Appl., 2013 (2013), 14 pages. 1 